\title{
On the Level
}

Michael E. Mullins

Published online: 1 October 2010

(C) American College of Medical Toxicology 2010

Keyword Scientific writing

A "level" is a carpenter's tool which simply and elegantly compares the orientation of an object to the horizon. The correct term for a measurable quantity of a substance in a specified volume or mass of a specimen is "concentration."

It is common in colloquial speech to discuss the level of an analyte in a specimen (e.g., "Let's check the acetaminophen level."); however, when writing about the outcome of the laboratory analysis, authors should use concentration to denote the finite result.

Example 1 (incorrect): "The acetaminophen level was $220 \mathrm{mcg} / \mathrm{mL}$."

Example 2 (correct): "The acetaminophen concentration was $220 \mathrm{mcg} / \mathrm{mL} . "$
This distinction is analogous to that between "fewer" and "less" (the former referring to a finite, measurable quantity, and the other referring to an indefinite amount) [1].

If authors will use level less often, editors and reviewers will make fewer suggestions to use concentration.

Conflicts of Interests None

\section{Reference}

1. Mycyk MB (2009) Fewer grammatical errors means less editing. J Med Toxicol. doi:10.1007/s13181-010-0059-9

M. E. Mullins $(\triangle)$

Division of Emergency Medicine,

Washington University School of Medicine,

Campus Box 8072, 660 S. Euclid Avenue,

Saint Louis, MO 63110, USA

e-mail: mullinsm@wusm.wustl.edu 